Giulio Orecchia $\mathbb{D}$

\title{
Erratum to: Semi-factorial nodal curves and Néron models of jacobians
}

Published online: 1 September 2017

\section{Erratum to: manuscripta math. DOI 10.1007/s00229-016-0895-2}

Proposition 5.20 is false as stated. This is due to the presence of an error in its proof: the last sentence of page 24, that reads "Then $\delta\left(\chi_{w_{0}}\right)+\chi_{w_{1}}$ is supported on the old vertices", is false. Here is how to rectify this.

1. We introduce a new notation.

Notation. Let $(G, l)=(V, E, l)$ be an $\mathbb{N}_{\infty}$-labelled graph. We let $\left(G, l^{\circ}\right)=$ $\left(V, E, l^{\circ}\right)$ be the $\mathbb{N}_{0}:=\mathbb{Z}_{\geq 0}$-labelled graph obtained from $(G, l)$ by setting $l^{\circ}(e)=$ 0 for all edges $e$ with label $l(e)=\infty$.

2. We replace Definition 5.19 by the following.

Definition. Let $(G, l)=(V, E, l)$ be an $\mathbb{N}_{\infty}$-labelled graph. We say that the labelled graph $(G, l)$ is circuit-coprime if for every circuit $C \subset G$, $\operatorname{gcd}\left(l^{\circ}(e) \mid e\right.$ is an edge of $\left.C\right)=1$. Here we impose that the gcd of a subset $S \subset \mathbb{Z}$ is the non-negative generator of the ideal $\langle S\rangle \subset \mathbb{Z}$.

3. Proposition 5.20 is then true as originally stated:

Let $(G, l)$ be an $\mathbb{N}_{\infty}$-labelled graph. The map (7) $H \rightarrow$ colim $H_{i}$ is an isomorphism if and only if $(G, l)$ is circuit-coprime.

Proof. For an $\mathbb{N}_{0}$-labelled graph instead of an $\mathbb{N}_{\infty}$-labelled one, we adopt the same notion of Cartier vertex-labelling and multidegree operator, by imposing that the only integer divisible by 0 is 0 and that $0 / 0=0$; then, letting $\left(G_{n}, l_{n}\right)_{n \in \mathbb{N}}$ be the blow-up graphs of $(G, l)$, the chain of homomorphisms (6) associated to $\left\{(G, l),\left(G_{n}^{\circ}, l_{n}^{\circ}\right)_{n \in \mathbb{N}}\right\}$ coincides with the one associated to $\left\{(G, l),\left(G_{n}, l_{n}\right)_{n \in \mathbb{N}}\right\}$. Also, we define the labelled circuit matrix $M_{\left(G, l^{\circ}\right)}$ and the labelled fundamental circuit matrix $N_{\left(G, l^{\circ}\right)}$ of an $\mathbb{N}_{0}$-labelled graph $\left(G, l^{\circ}\right)$ in the same way as for an $\mathbb{N}$-labelled one; then, Lemma 5.15 remains true for $\mathbb{N}_{0}$-labelled graphs, thus $(G, l)$ is circuit-coprime if and only if $N_{\left(G, l^{\circ}\right)}$ is surjective.

The online version of the original article can be found under doi:10.1007/s00229-016-08952.

G. Orecchia (凶): Mathematisch Instituut Leiden, Niels Bohrweg 1, 2333 CA Leiden, The Netherlands. e-mail: g.orecchia@math.leidenuniv.nl 
From now on we will work only with $\mathbb{N}_{0}$-labelled graphs, but we will drop the "o" to make the notation lighter.

The rest of the proof is now similar to the proof of Prop. 5.16. We want to show that $N_{(G, l)}$ is surjective if and only if $\epsilon_{n}: H \rightarrow H_{n}$ is surjective for all $n \geq 1$. We can fix an integer $n$ big enough, so that all labels of $G_{n}$ are 1's or 0 's. Let $\alpha \in \mathbb{Z}^{V_{n}}$; we may pick $\alpha=\chi_{v}$ for a vertex $v$ belonging to some path $P \subset G_{n}$ associated with some edge $\bar{e} \in E$. Denoting by $w_{0}, w_{1}, \ldots, w_{r}$ the vertices of the path $P$, we may assume that $v=w_{1}$ : if there is no edge in $P$ labelled by zero, the argument is the same as in the proof of Prop. 5.16; otherwise, there is only one such edge, connecting $w_{s}$ and $w_{s+1}$, with $s=\frac{r-1}{2}$. In this case, by possibly inverting the order of the numbering of the vertices of $P$, we first reduce to the case $v=w_{k}$ with $k \leq s$; then we have $\overline{\chi_{w_{k}}}=k \overline{\chi_{w_{1}}}$ similarly as in Prop. 5.16.

Now, a Cartier vertex-labelling $\widetilde{\phi}$ in $\mathcal{C}_{n}$ is such that $\delta_{n}(\widetilde{\phi})+\chi_{w_{1}}$ is supported on the old vertices if and only if for every new vertex $z$, adjacent to vertices $z_{1}$ and $z_{2}$,

$$
\begin{cases}\widetilde{\varphi}(z)-\widetilde{\varphi}\left(z_{1}\right)=\widetilde{\varphi}\left(z_{2}\right)-\widetilde{\varphi}(z) & \text { if } z \neq w_{1} \\ \left(\widetilde{\varphi}\left(z_{1}\right)-\widetilde{\varphi}(z)\right)+\left(\widetilde{\varphi}\left(z_{2}\right)-\widetilde{\varphi}(z)\right)=-1 & \text { if } z=w_{1}\end{cases}
$$

Note that by definition of a Cartier vertex-labelling, there exists $\widetilde{\varphi} \in \mathcal{C}_{n}$ satisfying (1) if and only if there exists a vertex-labelling $\widetilde{\phi} \in \mathbb{Z}^{V_{n}}$ satisfying (1) plus the extra condition that $\widetilde{\phi}\left(z_{1}\right)=\widetilde{\phi}\left(z_{2}\right)$ for any adjacent vertices $z_{1}, z_{2}$ connected by an edge labelled by 0 .

In turn, there exists such a $\widetilde{\varphi} \in \mathbb{Z}^{V_{n}}$ if and only if there exists $\phi \in \mathbb{Z}^{V}$ such that for every edge $e \in E$ with endpoints $v_{0}, v_{1}$

$$
\left\{\begin{array}{l}
\varphi\left(v_{1}\right)-\varphi\left(v_{0}\right) \equiv 0 \bmod l(e) \text { if } e \neq \bar{e} \\
\varphi\left(v_{1}\right)-\varphi\left(v_{0}\right) \equiv 1 \bmod l(e) \text { if } e=\bar{e}, v_{0}=w_{0}, v_{1}=w_{r}
\end{array}\right.
$$

where we have identified the old vertices $w_{0}, w_{r}$ with the corresponding vertices in $G$. Notice that being congruent modulo 0 means being equal, as the identity $\mathbb{Z} \rightarrow \mathbb{Z}$ is a quotient map with respect to equivalence modulo 0 . Now, condition (2) above is the same as condition (4) in Prop. 5.16., and from this point on the rest of the proof coincides with the proof of Prop. 5.16; we only mention that, at the point when $x(e)$ is defined, one can assign to it any value if $l(e)=0$.

Finally, we show that with the old version of Definition 5.19, Theorem 6.3 (which follows from Proposition 5.20) is false. Here is a counterexample: take $S=\operatorname{Spec}(k[[t]])$, and $X / S$ the constant family given by $y^{2}=x^{3}-x^{2}$. Then the labelled graph $(G, l)$ of the special fibre consists of a single loop labelled by $\infty$ and thus is circuit-coprime with respect to the old definition; however, $X / S$ is not semi-factorial. 